\title{
Protective effect of pyrroloquinoline quinine on ultraviolet $A$ irradiation-induced human dermal fibroblast senescence in vitro proceeds via the anti-apoptotic sirtuin 1/nuclear factor-derived erythroid 2-related factor $2 /$ heme oxygenase 1 pathway
}

\author{
CHUNLI ZHANG ${ }^{1}$, CHUANJUN WEN ${ }^{2},{\text { JINDE } \text { LIN }^{3} \text { and GAN SHEN }}^{3}$ \\ ${ }^{1}$ Department of Clinical Research, Friendship Plastic Surgery Hospital, Nanjing Medical University, Nanjing, Jiangsu 210029; \\ ${ }^{2}$ College of Science, Nanjing Normal University, Nanjing, Jiangsu 210046; ${ }^{3}$ Department of Plastic Surgery, \\ The Second Affiliated Hospital of Nanjing Medical University, Nanjing, Jiangsu 210000, P.R. China
}

Received May 20, 2014; Accepted February 13, 2015

DOI: $10.3892 / \mathrm{mmr} .2015 .3990$

\begin{abstract}
The aim of the present study was to determine whether pyrroloquinoline quinine (PQQ) exerts a protective effect on ultraviolet A (UVA) irradiation-induced senescence in human dermal fibroblasts (HDFs) and to elucidate its mechanism of action in vitro. A senescence model was constructed as follows: HDFs $\left(1 \times 10^{4}-1 \times 10^{6}\right)$ were cultured in a six-well plate in vitro and then exposed to UVA irradiation at a dosage of $9 \mathrm{~J} / \mathrm{cm}^{2}$. Various concentrations of PQQ (50, 100 and $200 \mathrm{ng} / \mathrm{ml}$ ) were added to the culture medium $24 \mathrm{~h}$ prior to UVA exposure. Following $72 \mathrm{~h}$ of irradiation, senescence-associated $\beta$-galactosidase staining was performed in order to evaluate the senescence state. Furthermore, mRNA expression of the senescence marker genes matrix-metalloprotease (MMP)1 and MMP3 was determined using reverse transcription quantitative polymerase chain reaction. Protein expression of sirtuin (SIRT)1, SIRT6, nuclear factor erythroid 2-related factor 2 (Nrf2) and heme oxygenase 1 (HO-1) were detected using western blot analysis. The results showed that the percentage of cells stained by X-gal following $9 \mathrm{~J} / \mathrm{cm}^{2} \mathrm{UVA}$ irradiation was markedly increased compared with that of the control group (53 and 8\%, respectively), while $50 \mathrm{ng} / \mathrm{ml}$ PQQ attenuated the ratio of positive staining compared with that of the UVA-only cells (29 vs. 53\%, respectively). Expression of fibroblast senescence marker genes MMP1 and MMP3 was decreased in cells treated with UVA and $50 \mathrm{ng} / \mathrm{ml}$ PQQ compared with that of cells in the UVA-only group. Western
\end{abstract}

Correspondence to: Mr. Gan Shen, Department of Plastic Surgery, The Second Affiliated Hospital of Nanjing Medical University, 262 North Zhongshan Road, Nanjing, Jiangsu 210000, P.R. China E-mail: ganshenmd@126.com

Key words: pyrroloquinoline quinine, ultraviolet A irradiation-induced senescence, sirtuin 1/nuclear factor-derived erythroid 2-related factor 2 /heme oxygenase 1 pathway blot analysis revealed significant effects of PQQ on SIRT1 and SIRT6. Nrf2 and HO-1 exbibited mild changes with the same trend when treated with or without UVA and PQQ. In conclusion, the results of the present study showed that pyrroloquinoline quinine may have a protective effect on UVA irradiation-induced HDF aging, which may be associated with the anti-apoptotic SIRT1/Nrf2/HO-1 pathway as well as SIRT6 signaling.

\section{Introduction}

Skin is an essential natural barrier to protect body from outside physical, chemical, microbial and other infestation; in addition, skin is a visual indicator of the body's aging process (1). $90 \%$ of the ultraviolet (UV) radiation reaching the surface of the earth is long-wavelength radiation (UVA; 320-400 $\mathrm{nm}$ ), which is 20 times higher than that of medium-wavelength radiation (UVB; 280-320 $\mathrm{nm}$ ), while short-wavelength radiation (UVC; $<290 \mathrm{~nm}$ ) is completely absorbed by the ozone shield (2). Under physiological conditions, UVA penetrates the epidermis into the dermis; therefore, it has been well-established that UVA irradiation is responsible for photoaging and photocarcinogenesis (3).

Pyrroloquinoline quinine (PQQ) is a non-covalently bound redox co-factor of bacterial dehydrogenases, which was initially isolated from cultures of methylotropic bacteria, and does not rely on nicotinamide adenine dinucleotide phosphate (NADP) and NAD as well as flavin adenine dinucleotide (FAD). It was later reported that PQQ was abundant in various types of plant as well as in milk, animals and humans. PQQ was therefore defined as a novel type of vitamin (4), which was found to have important roles in the promotion of growth, cell proliferation and growth factor secretion (5). Evidence has suggested that PQQ is also involved in redox processes in the mitochondrial respiratory chain, scavenging of reactive oxygen species (ROS), attenuation of oxidative stress in mitochondria (6) and the protection of neurons (7); in addition, PQQ was reported to antagonize several types of oxidative stress-induced cell damage, including reoxygenation injury in 
the heart, ethanol-induced liver damage and hyperoxia-induced cognitive deficits (8). The present study aimed to determine whether PQQ was involved in the protection of human dermal fibroblasts (HDFs) from UVA-induced senescence.

In order to investigate the protective mechanisms of $\mathrm{PQQ}$ against UVA-induced HDF aging, the present study assessed the expression of two members of the sirtuin (SIRT) family: SIRT1 and SIRT6. Nuclear and cytoplasm-localized SIRT1 has been reported to have important roles in apoptosis, differentiation and oncogenic transformation (9-14). SIRT1 was also found to deacetylate transcription factors and co-activators, such as heat shock factor 1 , which induces the transcription of molecular chaperones associated with the pathogenesis of Parkinson's disease and amyothrophic lateral sclerosis (15-20). Furthermore, SIRT6 was shown to be crucial in the regulation of mammalian longevity; therefore, it was hypothesized that SIRT6 may have potential for the treatment of age-associated diseases $(21,22)$.

The present study aimed to determine whether PQQ influences the damage of UVA irradiation, and how it functions in the cell. It was hypothesized that PQQ protects human dermal fibroblasts from senescence caused by UVA irradiation. An in vitro cell-senescence model was constructed through the exposure of PQQ-pre-treated HDFs to UVA, and the effect of PQQ on protein and/or mRNA expression levels of the senescence marker genes matrix metalloproteinase (MMP)1 and MMP3 as well as SIRT1, SIRT6, nuclear factor erythroid 2-related factor 2 (Nrf2) and heme oxygenase 1 (HO-1) were detected using polymerase chain reaction (PCR) and western blot analyses. Furthermore, senescence-associated $\beta$-galactosidase (SA- $\beta$-Gal) staining was used to determine the senescence status of HDFs (8). SA- $\beta$-Gal activity distinguishes senescent cells from those which are quiescent or terminally differentiated, therefore acting as a senescence biomarker.

\section{Materials and methods}

Cell culture. Primary HDFs were purchased from BioHermes Bio\& Medical Technology Co., Ltd (Wuxi, China). Fibroblasts were subsequently cultured in a $10-\mathrm{cm}$ dish in Dulbecco's modifed Eagle's medium (DMEM; Gibco Life Technologies, Carlsbad, CA, USA) supplemented with $15 \%$ fetal bovine serum (FBS; Gibco Life Technologies), penicillin $(100 \mathrm{U} / \mathrm{ml})$ and streptomycin $(100 \mu \mathrm{g} / \mathrm{ml}$; Gibco Life Technologies). Cells were divided into aliquots and seeded into a six-well plate (Corning Incorporated, Corning, NY, USA), then incubated in an incubator (3111; Thermo Fisher Scientific, Waltham, MA, USA) at $37^{\circ} \mathrm{C}$ with $5 \% \mathrm{CO}_{2}$ in a humidified atmosphere. For cell culture, the medium was replaced every 2-3 days and fibroblasts were passaged at $80 \%$ confluence. Cells were then seeded in a 6 -well plate at a density of $1 \times 10^{6}$ cell $/ \mathrm{cm}^{2}$ for use in subsequent experiments.

UVA irradiation. At $24 \mathrm{~h}$ following the addition of PQQ (Sigma-Aldrich, St. Louis, MO, USA) into the culture media, cells in the six-well plate were exposed to $9 \mathrm{~J} / \mathrm{cm}^{2}$ UVA irradiation. Cells were washed with phosphate-buffered saline (PBS; Gibco Life Technologies) and covered with a thin layer of PBS prior to UVA exposure. The culture plate lid was removed, and the six-well plate was placed on a brass block embedded on ice, in order to reduce any evaporation, at a distance of $15 \mathrm{~cm}$ from the UVA light source. As the UVA irradiation source, an Ultraviolet phototherapy instrument (SS-04A; Shanghai SIGMA High-Tech Co., Ltd, Shanghai, China) equipped with a 15-W ozone-free UVA lamp (CEL015 W; Philips, Groningen, Holland) was used. The incidence dose of UVA was measured with a UVA/UVB-ultraviolet meter (Factory affiliated to Beijing Normal University, Beijing, China). Following exposure to UVA for varying lengths of time, PBS was replaced with culture medium and cells were incubated under standard conditions for $72 \mathrm{~h}$ prior to analysis.

Total RNA isolation. UVA irradiation and PQQ treatment were performed as described above. At $72 \mathrm{~h}$ post-irradiation, total RNA was extracted from the cells using TRIzol reagent (Promega Corp., Madison, WI, USA). RNA concentration and purity were determined using a Nanodrop-2000 UV spectrophotometer (Thermo Fisher Scientific). Ribosomal RNA band integrity was evaluated using conventional denaturing agarose gel electrophoresis (23) using the SDS-PAGE gel quick preparation kit (Beyotime Institute of Biotechnology, Shanghai, China).

Reverse transcription quantitative PCR (RT-qPCR). Samples were normalized to total RNA content prior to reference gene assessment and gene expression analysis. Equal amounts of RNA (300 ng) from each sample were reverse-transcribed using a PrimeScript ${ }^{\mathrm{TM}}$ RT Reagent kit with gDNA Eraser (Takara Bio, Inc, Dalian, China) according to manufacturer's instructions. qPCR was performed using SYBR green dye method (SYBR Premix Ex Taq; Takara Bio Inc.) using an ABI700 Real-Time PCR detection system (Applied Biosystems; Life Technologies, Thermo Fisher Scientific). Three biological and two technical replicates per biological sample were performed. The following standard cycling conditions for qPCR runs were applied: $95^{\circ} \mathrm{C}$ for $3 \mathrm{~min}$ to activate polymerase, 40 cycles of denaturation at $95^{\circ} \mathrm{C}$ for $15 \mathrm{sec}$ and annealing-extension at $60^{\circ} \mathrm{C}$ for $30 \mathrm{sec}$. Melting curve analysis was performed following every run by defined heating up to $95^{\circ} \mathrm{C}$ to assess the presence of unspecific PCR products. A negative control was included in each assay run, using water instead of template.

Specific primers for the RT-qPCR reaction were as follows: MMP-1 forward, 5'-TTGGAGGGGATGCTCATT-3' and reverse, 5'-ACACGCTTTGGGGTTTG-3' with a product size of $103 \mathrm{bp}$; MMP3 forward, 5'-GCAGTTTGATCAGCC TATCC-3' and reverse, 5'-TCCAGAGTGTCGGAGTCCAG-3' with a product size of $138 \mathrm{bp}$; SIRT1 forward, 5'-TGACTGTGA AGCTGTACGAGGAG-3' and reverse, 5'-GGAAGACCCAAT AACAATGAGGA-3' with a product size of $120 \mathrm{bp}$; SIRT6 forward, 5'-CCCACGGAGTCTGGACCAT-3' and reverse, 5'-CTCTGCCAGTTTGTCCCTG-3' with a product of $194 \mathrm{bp}$; $\beta$-actin forward, 5'-TGGAATCTTGCTCTTATTTTCACA-3' and reverse, 5'-TAAAACGCAGCTCAGTAACAGTCCG-3'.

All primers were synthesized by Sangon Biotech, Co., Ltd. (Shanghai, China) and were used at $400 \mathrm{nM}$ except for $\beta$-actin at $300 \mathrm{nM}$. All PCR efficiencies were between 90 and $110 \%$. Expression data of reference genes were validated using geNorm 3.5 software (http://medgen.ugent.be/genorm/), as previously described (24). 
A
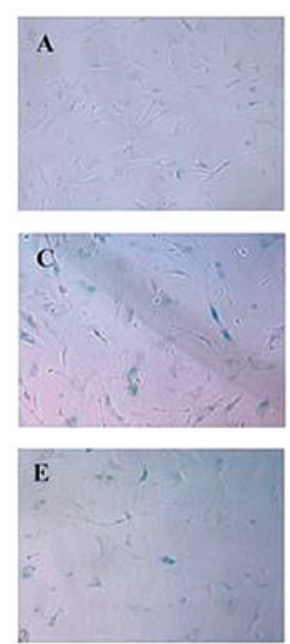
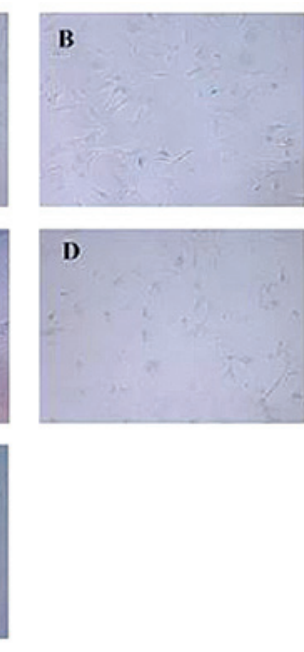

B

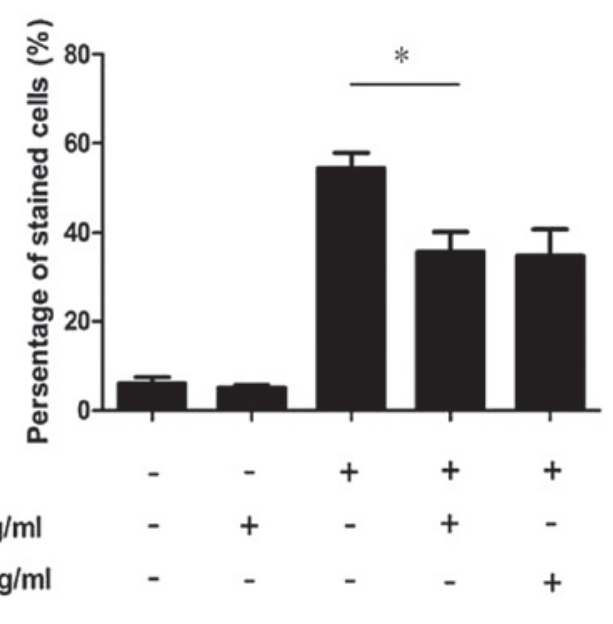

Figure 1. $\beta$-Galactosidase expression in HDFs following treatment with UVA and/or PQQ. (A) Representative images of HDFs treated as follows: a) Untreated, b) $50 \mathrm{ng} / \mathrm{ml} \mathrm{PQQ,} \mathrm{c)} 9 \mathrm{~J} / \mathrm{cm}^{2} \mathrm{UVA}$ irradiation, d) $50 \mathrm{ng} / \mathrm{ml} \mathrm{PQQ} \mathrm{prior} \mathrm{to} 9 \mathrm{~J} / \mathrm{cm}^{2} \mathrm{UVA}$ irradiation, e) $100 \mathrm{ng} / \mathrm{ml}$ PQQ prior to $9 \mathrm{~J} / \mathrm{cm}^{2} \mathrm{UVA}$ irradiation (magnification, x20). Staining was performed $72 \mathrm{~h}$ after irradiation. (B) Quantitative analysis of the percentage of cells positive for $\beta$-galactosidase activity following staining with senescence-associated- $\beta$-galactosidase. Values are presented as the mean \pm standard deviation ( $\mathrm{n}=3$ ). ${ }^{*} \mathrm{P}<0.05$, compared with $\mathrm{HDFs}$ treated with UVA only. HDFs, human dermal fibroblasts; UVA, ultraviolet A; PQQ, pyrroloquinoline quinine.

A

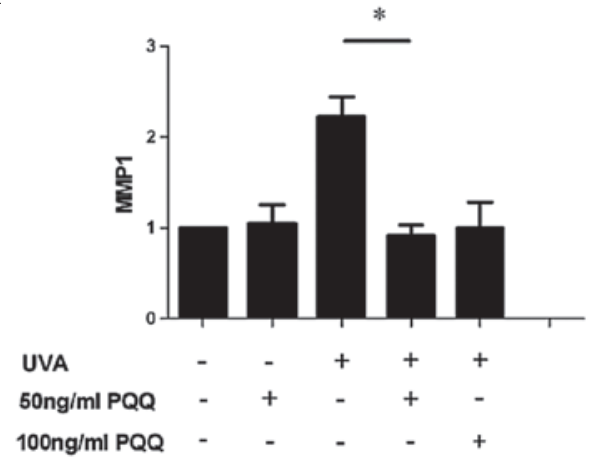

B

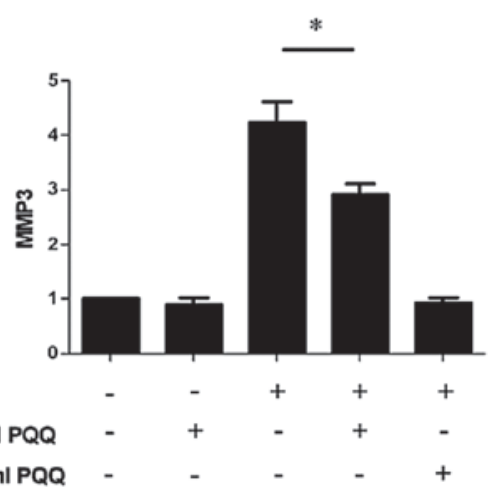

Figure 2. PQQ reduces the expression of senescence marker genes MMP1 and MMP3. Human dermal fibroblasts were irradiated with $9 \mathrm{~J} / \mathrm{cm}^{2} \mathrm{UVA}$ with or without pretreatment of 50 or $100 \mathrm{ng} / \mathrm{ml}$ PQQ and reverse transcription quantitative polymerase chain reaction was used to detect mRNA levels of (A) MMP1 and (B) MMP3. Total RNA was extracted $72 \mathrm{~h}$ after irradiation. Values are presented as the mean \pm standard deviation $(\mathrm{n}=3)$. " $\mathrm{P}<0.05$. $\mathrm{PQQ}$, pyrroloquinoline quinine; UVA, ultraviolet A; MMP, matrix metalloproteinase.

SA- $\beta$-Gal staining. SA- $\beta$-Gal activity was evaluated using a $\beta$-galactosidase staining kit (Beyotime Institute of Biotechnology, Haimen, China). Cells were washed with PBS and then fixed for $15 \mathrm{~min}$ at room temperature with fixative solution. The HDF cells were then incubated at $37^{\circ} \mathrm{C}$ overnight. SA- $\beta$-Gal-positive staining is expressed as a percentage of the total number of cells; cell numbers were counted in four continuous visual fields using a microscope (Olympus CX51; Olympus, Tokyo, Japan; total magnification, x20).

Western blot analysis. Whole-cell lysate extracts were separated using $12 \%$ SDS-PAGE, transferred to polyvinylidene difluoride membranes (Roche Diagnostics, San Francisco, CA, USA), blocked with 5\% bovine serum albumin (Sigma-Aldrich) and detected using the following primary antibodies: SIRT1 (mouse monoclonal IgG; cat. no. SC-74504; Santa Cruz Biotechnology, Inc., Dallas, TX, USA), SIRT6 (rabbit monoclonal IgG; cat. no. S4322; Santa Cruz Biotechnology, Inc.), Nrf2 (rabbit monoclonal IgG; cat. no. SC-365949; BD Biosciences, San Jose, CA, USA), HO-1 (mouse monoclonal IgG; cat. no. 610712; BD Biosciences) and GAPDH (mouse monoclonal $\mathrm{IgG}$; cat. no. G8795; Sigma-Aldrich), at a dilution of $1: 1,000$ and at $4^{\circ} \mathrm{C}$ for $8 \mathrm{~h}$. Horseradish peroxidase-conjugated goat anti-mouse and anti-rabbit immunoglobulin $\mathrm{G}$ were used as secondary antibodies at a dilution of 1:5,000 (cat. no. A3682; Sigma-Aldrich) at room temperature for $2 \mathrm{~h}$. GAPDH was used as an internal control. Blots were analyzed using an Super Signal West Pico Enhanced Chemiluminisence western blot analysis system (Thermo Fisher Scientific). Experiments were performed three times under identical conditions.

Statistical analysis. All statistical analyses were performed using GraphPad Prism software (GraphPad Inc., La Jolla, CA, USA). Values are presented as the mean \pm standard deviation. The one-way analysis of variance was used for comparisons involving more than two groups. $\mathrm{P}<0.05$ was considered to indicate a statistically significant difference between values. 


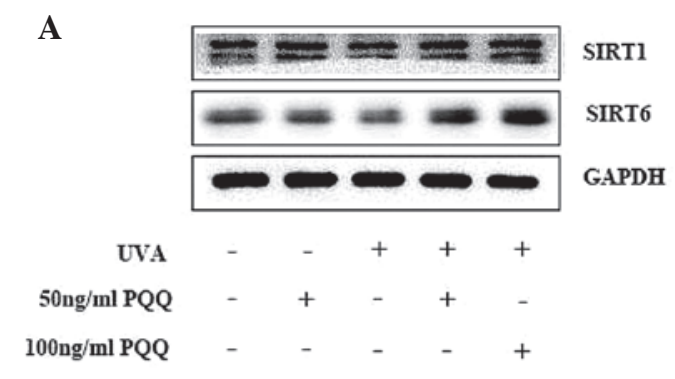

B

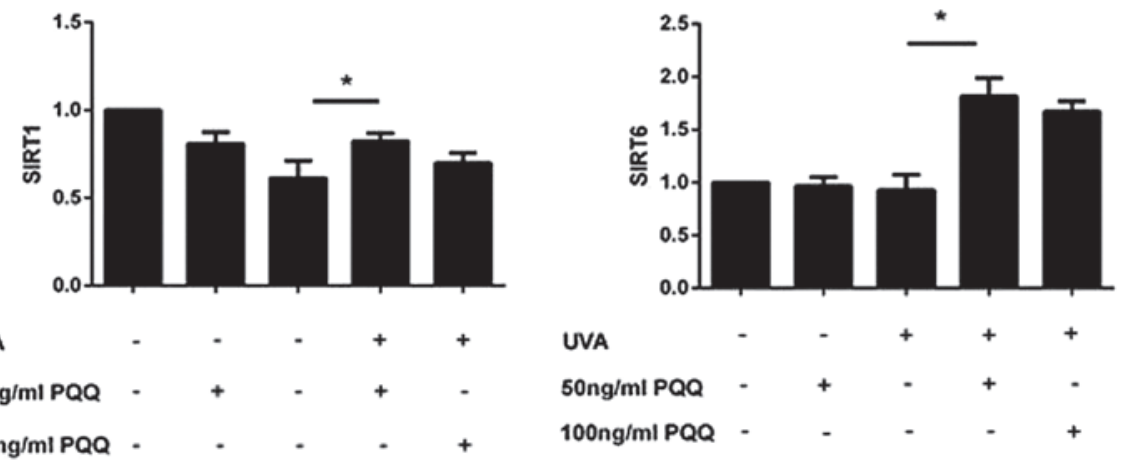

Figure 3. Low-dosage PQQ increases the expression of SIRT1 and SIRT6. (A) mRNA and (B) protein expression levels of SIRT1 and SIRT6 in human dermal fibroblasts irradiated with $9 \mathrm{~J} / \mathrm{cm}^{2} \mathrm{UVA}$ with or without pretreatment with 50 or $100 \mathrm{ng} / \mathrm{ml} \mathrm{PQQ}$. GADPH was used as an internal control. Protein was extracted $72 \mathrm{~h}$ after the UVA irradiation. Values are presented as the mean \pm standard deviation $(\mathrm{n}=3)$. " $\mathrm{P}<0.05$. PQQ, pyrroloquinoline quinine; UVA, ultraviolet A; SIRT, sirtuin.

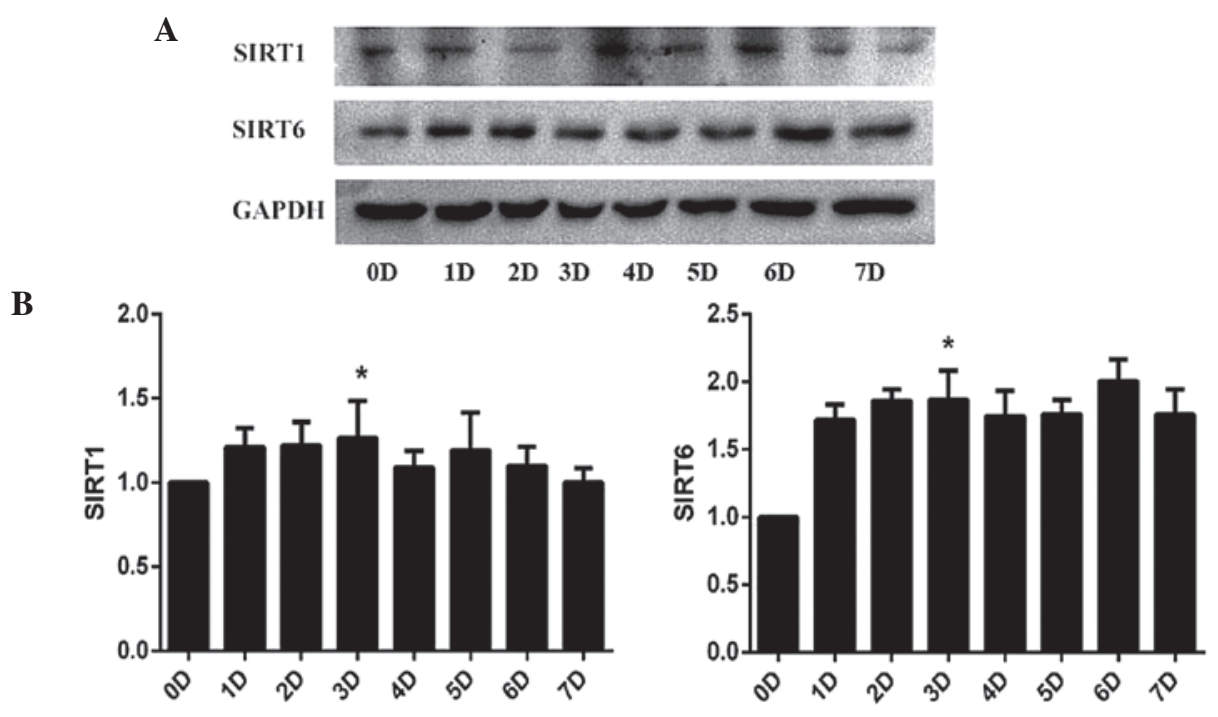

Figure 4. Expression pattern of SIRT1 and SIRT6 in UVA-irradiated HDFs pretreated with PQQ over seven days. (A) mRNA and (B) protein expression levels of SIRT1 and SIRT6 in HDFs irradiated with $9 \mathrm{~J} / \mathrm{cm}^{2}$ UVA with or without pre-treatment of $50 \mathrm{or} 100 \mathrm{ng} / \mathrm{ml} \mathrm{PQQ} \mathrm{were} \mathrm{recorded} \mathrm{daily} \mathrm{for} \mathrm{seven} \mathrm{days.} \mathrm{GADPH}$ was used as an internal control. Values are presented as the mean \pm standard deviation $(n=3)$. " $\mathrm{P}<0.05$. Total RNA and protein were extracted $72 \mathrm{~h}$ after the UVA irradiation. PQQ, pyrroloquinoline quinine; SIRT, sirtuin; UVA, ultraviolet A; HDFs, human dermal fibroblasts.

\section{Results}

Low-dose PQQ reduces $S A-\beta$-Gal activity in UVA-induced senescent HDFs. HDFs were pre-treated with various concentrations of PQQ (50 and $100 \mathrm{ng} / \mathrm{ml}$ ) and then subjected to UVA irradiation in order to induce senescence, which was detected using an SA- $\beta$-Gal staining kit. The results showed that the percentage of cells stained by X-gal following $9 \mathrm{~J} / \mathrm{cm}^{2}$ UVA irradiation was markedly increased compared with that in the control group (53 and $8 \%$, respectively; $\mathrm{P}<0.05$ ), while $50 \mathrm{ng} / \mathrm{ml}$ PQQ attenuated the ratio of positive staining compared with that of the UVA-only cells ( 29 vs. $53 \%$, respectively; $\mathrm{P}<0.01$ ) (Fig. 1A and B). In addition, the percentage of positively stained cells following treatment with $100 \mathrm{ng} / \mathrm{ml}$ PQQ and UVA were comparable to those of the cells treated with $50 \mathrm{ng} / \mathrm{ml} \mathrm{PQQ}$ and UVA (Fig. 1A and B).

Low-dose $P Q Q$ reduces the expression of senescence markers $M M P 1$ and MMP3 in UVA-irradiated HDFs. RT-qPCR analysis showed that mRNA expression levels of MMP1 and 
A

B

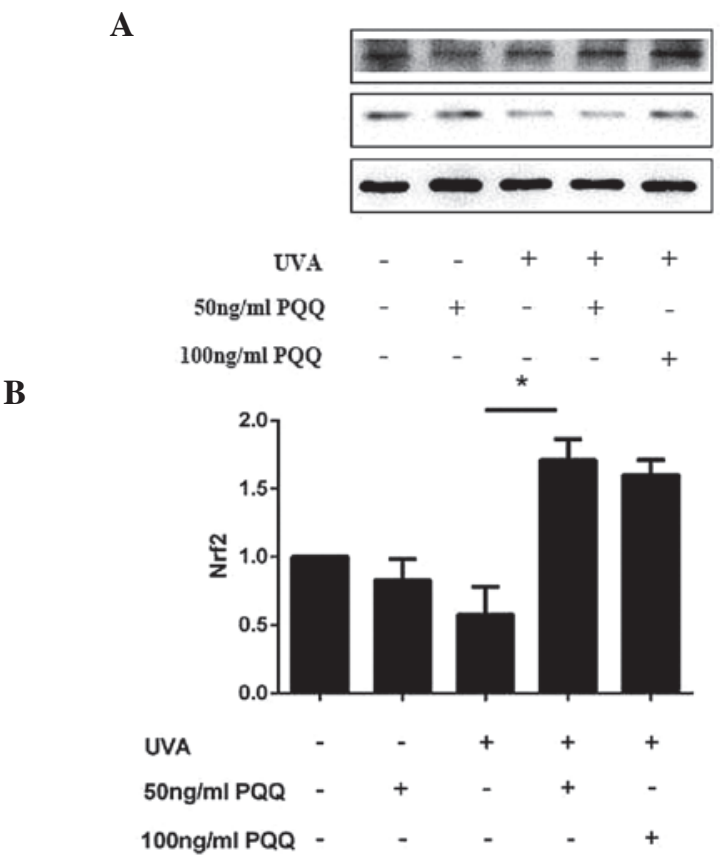

Nrf2
HO-1
GAPDH

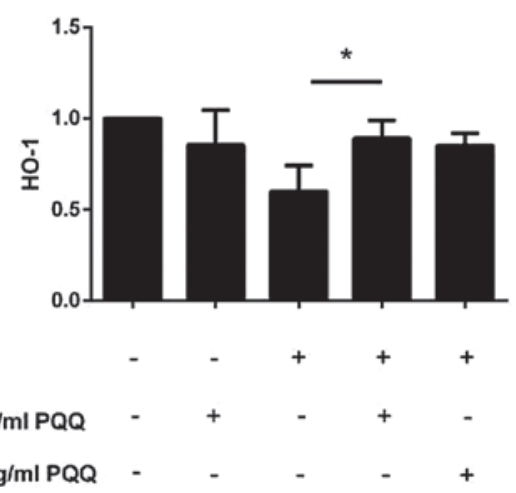

Figure 5. Low-dosage PQQ affects the expression of Nrf2 and HO-1 in UVA-irradiated HDFs. (A) mRNA and (B) protein expression of Nrf2 and HO-1 in HDFs irradiated with $9 \mathrm{~J} / \mathrm{cm}^{2}$ UVA with or without pre-treatment with 50 or $100 \mathrm{ng} / \mathrm{ml}$ PQQ. PQQ, pyrroloquinoline quinine; Nrf2, nuclear factor-derived erythroid 2-related factor 2; HO-1, heme oxygenase 1; UVA, ultraviolet A; HDFs, human dermal fibroblasts.

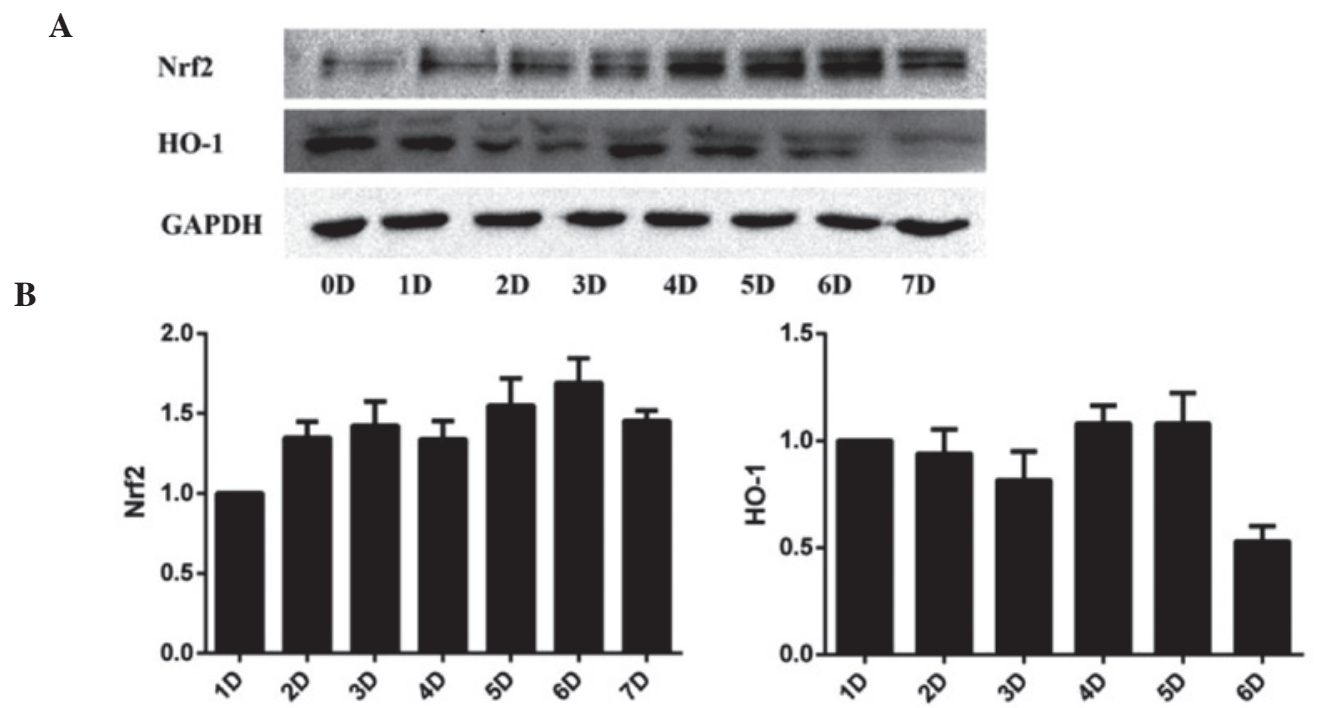

Figure 6. Expression pattern of Nrf2 and HO-1 in UVA-irradiated HDFs pretreated with PQQ over seven days. (A) mRNA and (B) protein expression levels of Nrf2 and HO-1 in HDFs irradiated with $9 \mathrm{~J} / \mathrm{cm} 2$ UVA with or without pretreatment of $50 \mathrm{or} 100 \mathrm{ng} / \mathrm{ml}$ PQQ were recorded daily for seven days. GADPH was used as an internal control. Values are presented as the mean \pm standard deviation $(\mathrm{n}=3)$. PQQ, pyrroloquinoline quinine; Nrf2, nuclear factor-derived erythroid 2-related factor 2; HO-1, heme oxygenase 1; UVA, ultraviolet A; HDFs, human dermal fibroblasts.

MMP3 were markedly reduced in UVA-irradiated HDFs pre-treated with 50 or $100 \mathrm{ng} / \mathrm{ml} \mathrm{PQQ}$ as compared with those in the UVA-only group $(\mathrm{P}<0.05)$ (Fig. 2).

SIRT1 and SIRT6 expression levels are increased in $U V A$-irradiated HDFs following pre-treatment with $P Q Q$. At $72 \mathrm{~h}$ following the addition of $50 \mathrm{ng} / \mathrm{ml} \mathrm{PQQ}$ to the culture media of UVA-irradiated HDFs, mRNA and protein expression levels of SIRT1 and SIRT6 were found to be increased (Fig. 3). In addition, notable differences were observed in the protein levels of SIRT1 and SIRT6 at $72 \mathrm{~h}$ following UVA irradiation.
In order to further study the efficacy of PQQ, western blot analysis was used to evaluate the effect of different concentrations of PQQ on SIRT1 and SIRT1 protein expression in UVA-irradiated HDFs. The results showed that $50 \mathrm{ng} / \mathrm{ml}$ and $100 \mathrm{ng} / \mathrm{ml}$ PQQ had a significant effect on SIRT1 and SIRT6 levels. Therefore, $50 \mathrm{ng} / \mathrm{ml}$ PQQ was used for the subsequent evaluation of the effect of PQQ on UVA-irradiation-induced HDF senescence at numerous time-points $(0,1,2,3,4,5,6$ and 7 days). The results showed that $50 \mathrm{ng} / \mathrm{ml}$ PQQ increased mRNA and protein expression of SIRT1 and SIRT6 within 72 h. $50 \mathrm{ng} / \mathrm{ml} \mathrm{PQQ}$ was added at the 5th day (Fig. 4). 
$P Q Q$ may activate the Nrf2/HO-1 pathway in UVA-irradiated HDFs. As SIRT1/Nrf2/HO-1 is a classical anti-apoptotic pathway $(11,23,24)$, the present study investigated whether this pathway was involved in UVA-induced cell apoptosis. Altered expression levels of Nrf2 and HO-1 mRNA expression levels were not found to be significant in HDFs pre-treated with $50 \mathrm{ng} / \mathrm{ml}$ PQQ without UVA irradiation. However, following UVA irradiation, PQQ was shown to alter the mRNA and protein expression of Nrf2 and HO-1 (Fig. 5A and B; Fig. 6). Of note, $50 \mathrm{ng} / \mathrm{ml}$ PQQ achieved the most significant effect on the expression of Nrf2 and HO-1 within $72 \mathrm{~h}$.

\section{Discussion}

In a recent study, PQQ was reported to act as a growth factor which contributed to mammalian growth and stimulated epithelial cells through the activation of epidermal growth factor receptor (25). PQQ has also been reported to protect primary cultured hippocampal neurons against glutamate-induced cell apoptosis, the mechanism of which was found to proceed via scavenging ROS and activating phosphatidylinositol 3-kinase/Akt/glycogen sythase kinase 3 $\beta / \mathrm{Nrf} 2$ signaling pathways (26). In addition, PQQ was reported to attenuate oxidative stress-induced cell damage in the heart, liver and brain (27). In the present study, a photoaging model was constructed in order to study the protective effects of PQQ on UVA-induced HDF senescence as well as the mechanisms by which this proceeds. The results demonstrated that low-dose PQQ protected HDFs from senescence following UVA irradiation, which may be associated with the anti-apoptotic Nrf2/HO-1 and SIRT1 pathways.

Fibroblasts are the primary cellular components of the human dermis and reflect the senescence status of skin at the cellular level (23). Long-wavelength UV radiation penetrates the epidermis to reach the dermis, where it exacerbates the photoaging of skin (21). In the present study, it was demonstrated that exposure of HDF to UVA under non-toxic conditions increased the mRNA and protein levels of MMP1 and MMP3. In addition, the expression of SA- $\beta$-Gal, a biomarker of fibroblasts in vivo and in vitro (8), was significantly upregulated in UVA-irradiated HDFs. However, PQQ reduced the expression of SA- $\beta$-Gal as well as mRNA expression levels of MMP1 and MMP3. These findings suggested that PQQ has a protective effect on cultured HDFs against UVA radiation.

Sirtuins (Sir) are class III deacetylases, a family of proteins that require NAD as a co-factor; therefore, they are controlled by cellular [NAD]/[NADH] ratios and respond to changes in the cellular metabolism (8), which is essential for lifespan extension by calorie restriction in yeast, worms and flies (28). Seven Sir2 homologs (SIRT1-7) have been identified in mammals and have important roles in the regulation of oxidative stress, DNA damage and metabolism, therefore making them good candidates as lifespan regulators (29). PQQ has been shown to activate cyclic adenosine monophosphate response factors, including Nrf1, Nrf2 and mitochondrial transcription factors to enhance mitochondriogenesis (30-33). The present study demonstrated that mRNA and protein expression of SIRT1, SIRT6 and Nrf2 were increased in a group of HDFs treated with $50 \mathrm{ng} / \mathrm{ml}$ PQQ and UVA radiation; of note, improvements were observed within $72 \mathrm{~h}$. In addition, decreased mRNA and protein expression of SIRT1, SIRT6, Nrf2 and HO-1 following UVA treatment were partially recovered when treated with $50 \mathrm{ng} / \mathrm{ml}$ PQQ. This observation indicated that Nrf2/HO-1 signaling may have an important role in the protective effect of PQQ against UVA irradiation-induced senescence in HDFs. As the increased expression of SIRT1, Nrf2 and HO-1 showed significant consistency, it was suggested that the SIRT1/Nrf2/HO-1 pathway was activated following PQQ treatment in UVA-irradiated HDFs.

In conclusion, the results of the present study demonstrated that PQQ protected HDFs from UVA irradiation in vitro, as shown by the upregulation of SIRT1 and SIRT6 following treatment with PQQ. In addition, the Nrf2/HO-1 signaling pathway was activated. The present study provided experimental evidence for the use of PQQ as a drug to prevent skin cell senescence and aging.

\section{Acknowledgements}

The present study was supported by the Nanjing Medical Science and Technology Development Fund Project (grant no. 2011NJMU242).

\section{References}

1. Kohl E, Steinbauer J, Landthaler M and Szeimies RM. Skin aging. J Eur Acad Dermatol 25: 873-884, 2011.

2. He YY, Council SE, Feng L and Chignell CF: UVA-induced cell cycle progression is mediated by a disintegrin and metalloprotease/epidermal growth factor receptor/AKT/Cyclin D1 pathways in keratinocytes. Cancer Res 68: 3752-3758, 2008.

3. Krutmann J: Ultraviolet A radiation-induced biological effects in human skin: relevance for photo aging and photodermatosis. J Dermatol Sci 23 (Suppl 1): S22-S26, 2000.

4. Salisbury SA, Forrest HS, Cruse WB and Kennard O: A novel coenzyme from bacterial primary alcohol dehydrogenases. Nature 280: 843-844, 1979.

5. Felton LM and Anthony C: Biochemistry: role of PQQ as a mammalian enzyme cofactor? Nature 433: E10-E12, 2005.

6. Matsushitak K, Toyama H, Yamada M, et al: Quinoproteins: structure function and biotechnologies applications. Appl Microboil Biot 58: 13-22, 2002.

7. Liu CL, Siesjö BK and Hu BR: Pathogenesis of hippocampal neuronal death after hypoxia-ischemia changes during brain development. Neuroscience 127: 113-123, 2004.

8. Dimri GP, Lee X, Basile G, et al. A biomarker that identifies senescent human cells in culture and in aging skin in vivo. Cell Biology 92: 9363-9367, 1995.

9. Ohwada K, Takeda H, Yamazaki M, Isogai H, Nakano M, Shimomura M, Fukui K and Urano S: Pyrroloquinoline quinone (PQQ) prevents cognitive deficit caused by oxidative stress in rats. J Clin Biochem Nutr 42: 29-34, 2008.

10. Zhang Q, Shen M, Ding M, Shen D and Ding F: The neuroprotective action of pyrroloquinoline against glutamate-induced apoptosis in hippocampal neurons is mediated through the activation of PI3K/Akt pathway. Toxicol Appl Pharm 252: 62-72, 2011.

11. Kanfi Y, Naiman S, Amir G, et al: The sirtuin SIRT6 regulates lifespan in male mice. Nature 483: 218-221, 2012.

12. Hekimi S and Guarente L: Genetics and the specificity of the aging process. Science 299: 1351-1354, 2003.

13. Howitz KT, Bitterman KJ, Cohen HY, et al: Small molecule activators of sirtuins extend Saccharomyces cerevisiae lifespan. Nature 425: 191-196, 2003.

14. Landry J, Sutton A, Tafrov ST, et al: The silencing protein SIR2 and its homologs are NAD-dependent protein deacetylases. Proc Natl Acad Sci USA 97: 5807-5811, 2000.

15. Sugino T, Maruyama M, Tanno M, et al: Protein deacetylase SIRT1 in the cytoplasm promotes nerve growth factor-induced neurite outgrowth in PC12 cells. FEBS Lett 584: 2821-2826, 2010 . 
16. Hisahara S, Chiba S, Matsumoto $\mathrm{H}$, et al: Histone deacetylase SIRT1 modulates neuronal differentiation by its nuclear translocation. Proc Natl Acad Sci USA 105: 15599-15604, 2008.

17. Michán S, Li Y, Chou MM, et al: SIRT1 is essential for normal cognitive function and synaptic plasticity. J Neurosci 30 9695-9707, 2010.

18. Jin Q, Yan T, Ge X, Sun C, Shi X and Zhai Q: Cytoplasm-localized SIRT1 enhances apoptosis. J Cell Physiol 213: 88-97, 2007.

19. Byles V, Chmilewski LK, Wang J, et al: Aberrant cytoplasm localization and protein stability of SIRT1 is regulated by PI3K/IGF-1R signaling in human cancer cells. Int J Biol Sci 6: 599-612, 2010.

20. Herskovits AZ and Guarente L: Sirtuin deacelylase in neurodegenera- tive disease of aging. Cell Res 23: 746-758, 2013.

21. Herrmann G, Brenneisen P, Wlaschek M, et al: Psoralen photoactivation promotes morphological and functional changes in fibroblasts in vitro reminiscent of cellular senescence. J Cell Sci 111: 759-767, 1998.

22. Brugè F, Venditti E, Tiano L, Littarru GP and Damiani E: Reference gene validation for qPCR on normoxia- and hypoxia-cultured human dermal fibroblasts exposed to UVA: is $\beta$-actin a reliable normalize for photoaging studies? J Biotechnol 156: 153-162, 2011

23. Kawahara TL, Michishita E, Adler AS, et al: SIRT6 links histone H3 lysine 9 deacetylation and organismal life span. Cell 136 62-74, 2008.

24. Herrmann G, Wlaschek M, Lange TS, et al: UVA irradiation stimulates the synthesis of various matrix-metalloproteinases (MMPs) in cultured human fibroblasts. Exp Dermatol 2: 92-97, 1993.

25. Masek T, Vopalensky V, Suchomelova P and Pospisek M: Denaturing RNA electrophoresis in TAE agrose gels. Anal Biochem 336: 46-50, 2005.
26. Vandesompele J, De Preter K, Pattyn F, et al: Accurate normalization of real-time quantitative RT-PCR data by geometric average of multiple internal control genes. Genome Biol 3: RESEARCH0034, 2002.

27. Li H, Wu S, Shi N, Lian S and Lin W: Nrf2/HO-1 pathway activation by manganese is associated with reactive oxygen species and ubiquitin-proteasome pathway, not MAPKs signaling. J Appl Toxicol 31: 690-697, 2011.

28. Kimura K, Takada M, Ishii T, et al: Pyrroloquinoline quinine stimulates epithelial cell proliferation by activating epidermal growth factor receptor through redox cycling. Free Radical Bio Med 53: 1239-1251, 2012.

29. Zhang Q, Ding M, Cao Z, et al: Pyrroloquinoline quinine protects rat brain cortex against acute glutamate-induced neurotoxicity. Neutovhrm Res 38: 1661-1671, 2013.

30. Li HH, He B, Peng H and Liu SQ: Effects of pyrroloquinoline quinine on proliferation and expression of c-fos, c-jun, CREB and PCNA in cultured Schwann cells. Zhonghua Zheng Xing Wai Ke Za Zhi 27: 298-303, 2011 (In Chinese).

31. Blander $G$ and Guarente L: The Sir family of protein deaceylases. Annu Rev Biochem 73: 417-435, 2004.

32. Lin SJ, Defossez PA and Guarente L: Requirement of NAD and SIR2 for life-span extension by calorie restriction in Sacharomyces cerevisiae. Science 289: 2126-2128, 2000.

33. Frye RA: Characterization of five human cDNAs with homology to the yeast SIR2 gene: Sir2-like proteins (sirtuins) metabolize NAD and may have protein ADP-ribosyltransferase activity. Biochem Biophys Res Commun 260: 273-279, 1999. 